\title{
How Many CMEs Have Flux Ropes? Deciphering the Signatures of Shocks, Flux Ropes, and Prominences in Coronagraph Observations of CMEs
}

\author{
A. Vourlidas ${ }^{1}$ - B.J. Lynch ${ }^{2}$ - R.A. Howard ${ }^{1}$. \\ Y. $\mathbf{L i}^{2}$
}

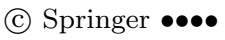

\begin{abstract}
We intend to provide a comprehensive answer to the question on whether all Coronal Mass Ejections (CMEs) have flux rope structure. To achieve this, we present a synthesis of the LASCO CME observations over the last sixteen years, assisted by 3D MHD simulations of the breakout model, EUV and coronagraphic observations from STEREO and SDO, and statistics from a revised LASCO CME database. We argue that the bright loop often seen as the CME leading edge is the result of pileup at the boundary of the erupting flux rope irrespective of whether a cavity or, more generally, a 3-part CME can be identified. Based on our previous work on white light shock detection and supported by the MHD simulations, we identify a new type of morphology, the 'two-front' morphology. It consists of a faint front followed by diffuse emission and the bright loop-like CME leading edge. We show that the faint front is caused by density compression at a wave (or possibly shock) front driven by the CME. We also present high-detailed multi-wavelength EUV observations that clarify the relative positioning of the prominence at the bottom of a coronal cavity with clear flux rope structure. Finally, we visually check the full LASCO CME database for flux rope structures. In the process, we classify the events into two clear flux rope classes ('3-part', 'Loop'), jets and outflows (no clear structure). We find that at least $40 \%$ of the observed CMEs have clear flux rope structures and that $\sim 29 \%$ of the database entries are either misidentifications or inadequately measured and should be discarded from statistical analyses. We propose a new definition for flux rope CMEs (FR-CMEs) as a coherent magnetic, twist-carrying coronal structure with angular width of at least $40^{\circ}$ and able to reach beyond $10 R_{\odot}$ which erupts on a time scale of a few minutes to several hours We conclude that flux ropes are a common occurrence in CMEs and pose a challenge for future studies to identify CMEs that are clearly not FR-CMEs.
\end{abstract}

Keywords: Coronal Mass Ejections, Low Coronal Signatures; Coronal Mass Ejections, Initiation and Propagation

\footnotetext{
1 Space Sciences Division, Naval Research Laboratory, Washington DC, USA

2 Space Sciences Laboratory, University of California, Berkeley, CA, USA
} 


\section{Introduction}

Since their detection in the early 1970s, Coronal Mass Ejections (CMEs) have been the subject of intense investigation with regard to their initiation mechanisms, their effects on the corona and their association with other coronal phenomena (eg., flares and prominences). This Topical Issue presents results from a Coordinated Data Analysis Workshop (CDAW) devoted to the question: 'Do All Coronal Mass Ejections (CMEs) Have Flux Rope Structures?' Such a specific physics-based question shows that we have come a long way towards understanding the nature of these explosive events especially when we consider the original definition of a CME: 'a relatively short scale white light feature propagating in a coronagraph's field of view' (paraphrasing Hundhausen et al., 1984).

Traditionally, CMEs were observed with visible light coronagraphs and clues on their origin and nature were based on their morphology in those images (Munro et al., 1979; Howard et al., 1985; Burkepile and St. Cyr, 1993). Despite the apparently large variation in the appearance of CMEs, two particular morphologies stand out: the 'loop'-CME where a bright narrow loop-like structure comprises the CME front, and the '3-part'-CME (Illing and Hundhausen, 1985) where the bright front is followed by a darker cavity which frequently contains a bright core. It has become the archetypical morphology of a CME even though the '3-part' morphology could be identified in only about a third of the events (Munro et al., 1979). It is still unclear whether the remaining variation is the result of projection effects due to the optically thin nature of the emission or not.

It was recognized early that the cavity rather than the prominence in the core drove the CME (Hundhausen, 1987). An initial controversy on whether CMEs were planar (i.e., ejected loops) or three-dimensional (i.e., bubbles) structures was largely resolved by the end of the 1980's. Crifo, Picat, and Cailloux (1983) demonstrated, using polarization analysis, that the loop front was indeed a bubble. The identification of halo CMEs by Howard et al. (1982) with their quasi-circular appearance established their three-dimensional (3D) nature and led to the adaption of the 'ice-cream' model to describe and fit the kinematics of these events (Howard et al., 1982, Zhao, Plunkett, and Liu, 2002, Xie, Ofman, and Lawrence, 2004: Xue, Wang, and Dou, 2005). A bubble or spherical structure is the intrinsic assumption behind this model which, by the way, is not a proper description as we will discuss later.

As theories progressed towards a more physical basis for the CME initiation, they focused on 3D magnetic topologies that could account for the '3-part' morphology and the frequent association with prominences. This quickly led to scenarios of rising loop arcades, overlying a prominence, which underwent reconnection to form magnetic flux ropes (FR, hereafter; Anzer and Pneuman, 1982 Forbes, 1990). Alternatively, the FR could pre-exist and rise under the driving of Lorenz forces (Kuperus and Raadu, 1974 Chen and Garren, 1993). While the question on whether the FR is formed before or during the eruption remains open, the overwhelming majority of magnetohydrodynamics (MHD) models and simulations agree on one thing. Namely, the erupting structure is 
always a FR (Chen, 2011). There is no physical mechanism that can produce a large-scale fast eruption from the corona without ejecting a fluxrope, to the best of our knowledge.

At the same time, in-situ measurements of interplanetary CMEs (ICMEs) often encounter structures with smooth rotation in one, or more, components of the magnetic field which can be fitted with FR models (Klein and Burlaga, 1982 Lepping, Burlaga, and Jones, 1990; Isavnin, Kilpua, and Koskinen, 2011, to name a few). These so-called Magnetic Clouds (MCs) can be considered then as the interplanetary manifestations of the ejected FR predicted by theory and possibly detected as the cavity in the '3-part'-CMEs (Burlaga et al., 1982). Cane and Richardson (2003) found that 100\% of ICMEs detected during solar minimum were MCs reducing to $<20 \%$ during solar maximum.

So the CDAW question regarding the nature of CMEs, at least in the case of ' 3 -part'-CMEs, seems to have been answered. A CME is simply the ejection of a magnetic FR structure from the lower corona which takes the form of a ' 3 part'-CME or a MC depending on the instrumenation used (images or in-situ, respectively) to detect it.

But, if a FR is a necessary ingredient for an ejection, why not all CMEs show evidence for such structure? In other words, why all CMEs are not '3-part'CMEs? Some have just a loop front while others appear as jets or structureless clouds or blobs. For example, Howard et al. (1985) categorized CMEs, between 6-10 $\mathrm{R}_{\odot}$, into ten morphological classes based on their appearance in Solwind observations. Why is there such a large variety of shapes? Could there be other types of magnetic structures, besides FRs, ejected from the Sun? If they do exist, they would suggest a major gap in our understanding of eruptive processes, given the prevalence of $\mathrm{FR}$ in our theories.

Second, not all ICMEs exhibit MC signatures. Is this simply a result of 'glancing' cuts between in-situ instruments and the ICME? Or do CME FRs lose their coherence as they travel in the interplanetary space, through reconnection with the ambient solar wind for example (Dasso et al., 2007)?

Third, many fast ICMEs are driving a shock followed by a sheath of postshocked plasma. The resulting five-part ICME (shock, sheath, dense front, cavity, and dense plug) does not have a coronal counterpart. Where are the five-part CMEs or more precisely, where are the shock and sheath signatures in the coronagraph images? Shocks could deflect streamers and generally affect the ambient corona, ahead and at the flanks of a CME, thus creating complex brightness distributions in the images. Could such effects be responsible for misidentifications, and hence misinterpretations, of CME morphologies, kinematic profiles, and associations with structures in the low corona or the inner heliosphere?

Fourth, and related point, the emission processes in both low (EUV) and middle (white light) corona are optically thin resulting in images that are projections on the plane of sky (POS). Do these projections affect our ability to properly interpret observations and how can we account for them? We will address this problem throughout this paper.

The Large Angle and Spectrometric Coronagraph (LASCO; Brueckner et al. 1995) project has accumulated the largest and longest database of coronagraphic observations of CMEs since 1996. Spanning more than a complete solar cycle, it is 
reasonable to expect that events of every possible orientation, size, speed, mass, and morphologies have been captured. We should be in position to understand the role of projection effects on the images, identify the origin of the various features (CME or not) in a given LASCO image, and hence answer the question posed in this Topical Issue.

To accomplish this task comprehensively we have given this paper a relatively large scope. It represents a synthesis of the observational knowledge gained over the sixteen years of LASCO observations. In the following sections, we will provide: evidence for the FR structure within CME cavities (Section 2), evidence for the existence of white-light shock and tips on distinguishing the shock front from the CME front (Section 3), theoretical support for these interpretations using synthetic images from 3D MHD simulations (Section 4), observations that clarify the connection between prominence and erupting cavity (Section 5), and finally statistics on the occurence of '3-part' or more precisely FR-CMEs, along with a discussion on the constrains of event lists (Section 6). We discuss and conclude in Section 7.

We will support several of our predictions and conclusions by using twoviewpoint imaging afforded by the Sun-Earth Connection Coronal and Heliospheric Investigation (SECCHI; Howard et al. 2008) on-board the Solar TErrestial RElations Observatory (STEREO) (Kaiser et al., 2008). We will use the SECCHI observations as necessary but we want to focus on the single viewpoint from LASCO for two reasons. First, this article is part of a workshop devoted on the analysis of events observed with LASCO. Second, and more important, the STEREO mission has a finite lifetime. Budgetary and other concerns suggest that future observations (whether research or operationally oriented) will be obtained from a single vantage point. It is therefore crucial that future observers can interpret such single viewpoint observations accurately.

\section{Where is the Flux Rope? The 3-part CME}

As we noted, the 3-part morphology was identified since the early coronagraph observations. The prototypical event is an event similar to the CME in Figure 1. All three components can be readily identified in this snapshot (the movie is available online) which was constructed by dividing the original image with a long-term background to remove the effects of the F-corona but to avoid removal of the ambient electron corona. An inspection of the accompanying movie reveals that the brightness of the front originates from the pile-up of the overlying streamer material. The core has sufficient structure to identify it unambiguously to the pre-eruption prominence (we will not discriminate between the terms 'filament' and 'prominence' here since they both refer to the same structure). Note that the cavity, while not completely devoid of plasma, does contain less electrons (it is less bright) than its surroundings. These aspects have been noted before. The question here is where is the evidence that the cavity is (or contains) a flux rope like structure?

Let us focus on the concave upward features labeled as 'horns'. They seem to originate within the core and to outline the extent of the cavity. Such configuration is consistent with models of prominence suspension at the bottom of 
a coronal flux rope cavity (Low and Hundhausen, 1995). Plunkett et al. (2000) commented on the appearance of these 'horns' in EIT images before the eruption as an indication of the formation of the fluxrope which subsequently erupted and they also noted that the prominence lay at the trailing edge of the CME. Similar structures were observed by Wood et al. (1999) and Dere et al. (1999) and interpreted in a similar way as direct evidence of the FR nature of the CME cavity.

Although these features have been observed in many events since, their FR association does not seem to be widely recognized. This may be because the low densities within the cavity do not permit an easy visualization of the FR structure when only the lower part (the 'horns') are illuminated. The missing 'link' would be a 3-part CME where the cavity would be filled with sufficient amount of plasma to illuminate the full volume and structure of the FR. Figure 2 shows such an example. The event is associated with a slow eruption of a quiet Sun prominence from the northern hemisphere. The last traces of $304 \AA$ disappear from the Extreme Ultraviolet Imager (EUVI)-A field of view at 1:56 UT on November, 4. This is by far the clearest detection of an FR within a CME despite observations of thousands of events with LASCO. We believe that the rarity of such detections is due to four reasons: (i) the clearest signatures will appear at middle corona heights (say, $>7 \mathrm{R}_{\odot}$ ) where the background streamer emission is weaker and the CME has finished evolving (Vourlidas et al., 2010). (ii) the lower spatial resolution of the $\mathrm{LASCO} / \mathrm{C} 3$ coronagraph (it is about $4 \times$ coarser than the LASCO/C2) washes out some of the fine scale detail. (iii) the FR must have a large size along the line of sight (LOS) and (iv) the FR must be oriented almost exactly perpendicular to the POS to produce bright emission throughout the cavity. The highly structured core of the CME in the COR2-A image has an almost identical appearance in the EUVI-A $195 \AA$ images (not shown here) which suggests that most of the core material was at coronal temperatures $(\sim 1.4 \mathrm{MK})$ erasing thus any obvious connections to the prominence (see Robbrecht, Patsourakos, and Vourlidas (2009) for a very similar example). We will return to this point in Section 5 .

Even such clear observations would not convince probably the skeptics that the cavity and FR are the same structure. The images are 2D projections on the POS leaving some room for misinterpretation. A comparison to theoretical predictions is therefore required. The high sensitivity of the LASCO images enabled the first opportunity for a detailed comparison between observations and theroreticaly-derived 2D FR structures (Chen et al., 1997, 2000). Krall and St. Cyr (2006) extended these comparisons to a larger sample of LASCO '3-part'CMEs in an effort to extract some 3D information (aspect ratio, eccentricity) of the FR. Krall (2007) attempted to answer the same question as us by comparing statistical distributions of the width and the rate of occurrence of concaveupward structures (essentially 'horns' seen in visible light) from observations to synthetic FR images with satisfactory agreement. Extensive measurements of the geometric properties of many 3-part CMEs led Cremades and Bothmer (2004) to conclude that 3-part CMEs were not simply spherical bubbles but structures elongated along the axis parallel to photospheric neutral line in their source regions. These results were subsequently confirmed by forward modeling 


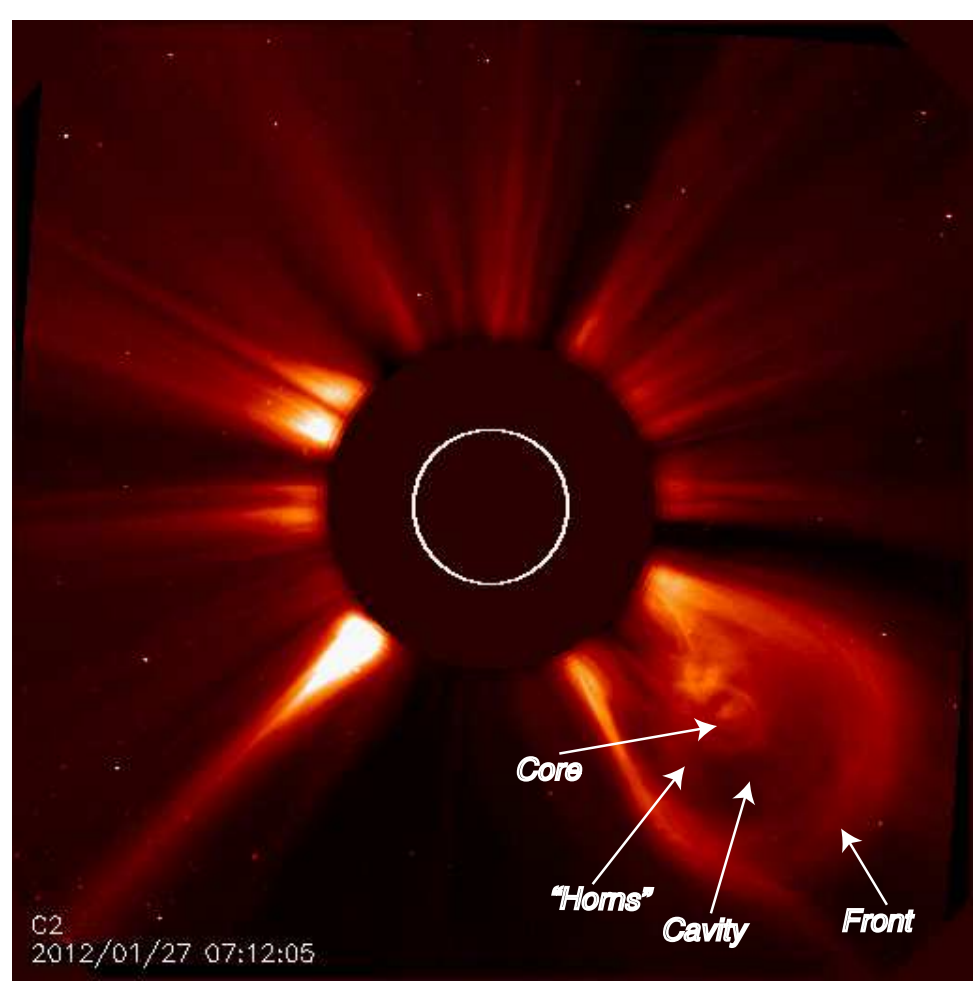

Figure 1. A typical 3-part CME as it appeared on LASCO/C2 on January 27, 2012. The three components are identified on this snapshot image. The full movie is available online.

methods which demonstrated that a 3D geometric representation of a FR-like shape could account for the observed CME density envelopes and shapes (Thernisien, Howard, and Vourlidas, 2006). This issue has now been consolidated with the successful application of FR-like geometric structures on stereoscopic observations from STEREO (Thernisien, Vourlidas, and Howard, 2009, 2011; Rouillard, 2011, Wood et al., 2011). There should be little doubt, therefore, that '3-part'-CMEs are indeed systems of ejected FRs where the cavity is the actual FR.

Our examples, and the events exhibiting clear 3-part structures in general, must lie close to the POS to provide an edge-on view of the FR. But CMEs occur in all longitudes. Occasionally, a '3-part'-CME will be observed along the SunEarth line. How can we then tell whether a halo CME has a 3-part structure and how can we identify the erupted FR against the backdrop of deflected streamers and material outflows? For this, we first need to identify the signatures of the other erupting structures starting with the shock.

\section{Where is the Shock? The 5-part CME}

It is common knowledge that sudden changes in plasma motion will launch compressive waves through the medium. If the motion occurs faster than the 


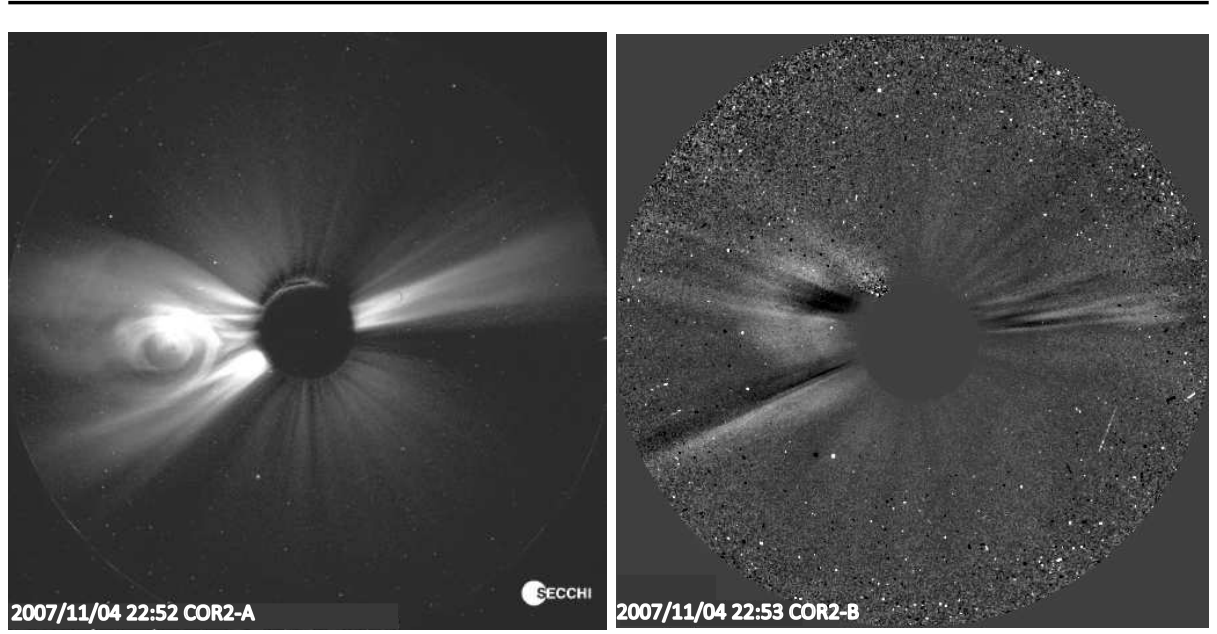

Figure 2. Left: An exceptionally clear example of flux rope structure entrained within a CME observed by SECCHI//COR2-A on November 11, 2007. There is no 'cavity' in this case since emission from the dense flux rope fills that region. Multiple striations including a tip at the backend of the structure can be discerned. The lack of a bright front is likely due to the low speed and high starting height for this event. Right: Simultaneous image of the same event from COR2-B. The CME appears as a partial halo and a base difference image is used to enhance the faint emission. There is no evidence of FR structure from this viewpoint.

characteristic speed of the medium (ie., sound speed for an unmagnetized plasma or fast-mode speed for a magnetized plasma) the waves will then steepen into shock waves. In either case, the propagaing wave will manifest itself as a propagating compression of density (and magnetic field where applicable). In our case, the propagating FR will generate a plasma wave which may look as another propagating front in a coronagraph (or EUV) image sequence. Depending on various factors, such as the impulsiveness and starting height of the event, and the magnetic and plasma configuration in the ambient corona, the density compression of the wave front could become strong enough to be detected (Vourlidas et al., 2003). Because waves (and shocks) are an intrinsic component of any eruption, their coronagraphic signatures have been the subject of debate since the first CME observations (see Vourlidas and Ontiveros (2009) for a historical discussion). It was generally accepted that distant streamer deflections were a reliable, but indirect, proxy for these waves (Gosling et al., 1974 Sheeley, Hakala, and Wang, 2000).

The first identification of the density enhancement from a CME-driven shock was reported by Vourlidas et al. (2003) thanks to the high sensitivity of the LASCO observations. Such signatures are now commonly reported in the literature (Yan et al., 2006; Ontiveros and Vourlidas, 2009: Vourlidas and Ontiveros, 2009 Gopalswamy et al., 2009 Bemporad and Mancuso, 2010; Vourlidas and Bemporad, 2012 Kim et al., 2012). We now know that the CME-induced waves can be detected in coronagraph images, that they are faint and, that they are located ahead of the FR front. So it should be straightforward to identify them in any image (assuming there is a reasonable expectation of a wave occurrence due to the speed of the CME, for example). Because these wave signatures are 
faint, the best approach is to use calibrated, excess mass images (to remove effects such as vignetting, background streamers, etc) and display them with high contrast.

An illustrative example is shown in the upper panels of Figure 3 where the same frame from a fast CME is shown with two different contrast ratios. On the left, the CME has the classical 3-part appearance with a very clear loop front. On the right, the higher contrast ratio allows to see a fainter front ahead which extends around the bright loop front and connects to the deflected streamer on the eastern flank. A series of other deflected streamers (or more likely substreamer structures) can be seen as radial striations occupying position angles from the deflected streamer, around the CME to the western equator. The faint front appears to be the outer envelope of these deflected streamers consistent with being a wave driven by the CME and propagating within a large-scale streamer. Two more examples of such fronts are shown in the bottom panels of Figure 3. On the left, a C2 image from a CME on June 11, 2000 shows a bright filamentary front (a Loop CME) preceeded by an extensive faint front connecting to a deflected streamer in the north. On the right, a C3 image shows again a faint front terminating at a deflected streamer but this time the front extends only to one side of the CME. No fronts, and tellingly no loop front either, are seen along the northern CME flank.

These images serve to illustrate our earlier point that the detectability of these shock fronts is highly dependent on the sensitivity of the observations. It is thus unsurprising (in retrospective) that such features have eluded detection in the pre-LASCO coronagraph experiments which lacked CCD detectors, large fields of view, and long-term uninterrupted observations. For many events, only the bright loop would be detected (Figure 3. top left) thus only allowing rather indirect and ambiguous arguments on the existence of a shock (Sime and Hundhausen, 1987).

A plausible criticism that may arise from our interpretation of these images is how can we be sure that the faint front is indeed related to density pile-up at a wave front and is not simply ejected material, i.e., coronal loops moving ahead of the CME in direct analogy to the bright front ahead of the cavity. This can be best answered by careful inspection of excess mass movies of these events. If the front in question is due to ejected material then a depletion should form behind it as it does behind the CME proper. If the front is caused by a wave, then the enhancement is due to density compression and not material transport, therefore no depletion should occur. The latter is the observed behavior for the events in Figure 3. MHD simulations have provided further support for this interpretation by matching the location and density compression ratio between observations and model (Vourlidas et al., 2003 Manchester et al., 2008). Recently, similar fronts have been detected in high cadence EUV images (Ma et al., 2011, Cheng et al., 2012).

Returning to the white light morphology, the identification of the faint front ahead of the bright loop-like CME front simplifies greatly the interpretation of CME images. The bright loop is the pile-up of material at the outer boundary of the erupted FR (the cavity) and hence it is bright while the outer front originates from a temporary compression of the ambient plasma as the wave 

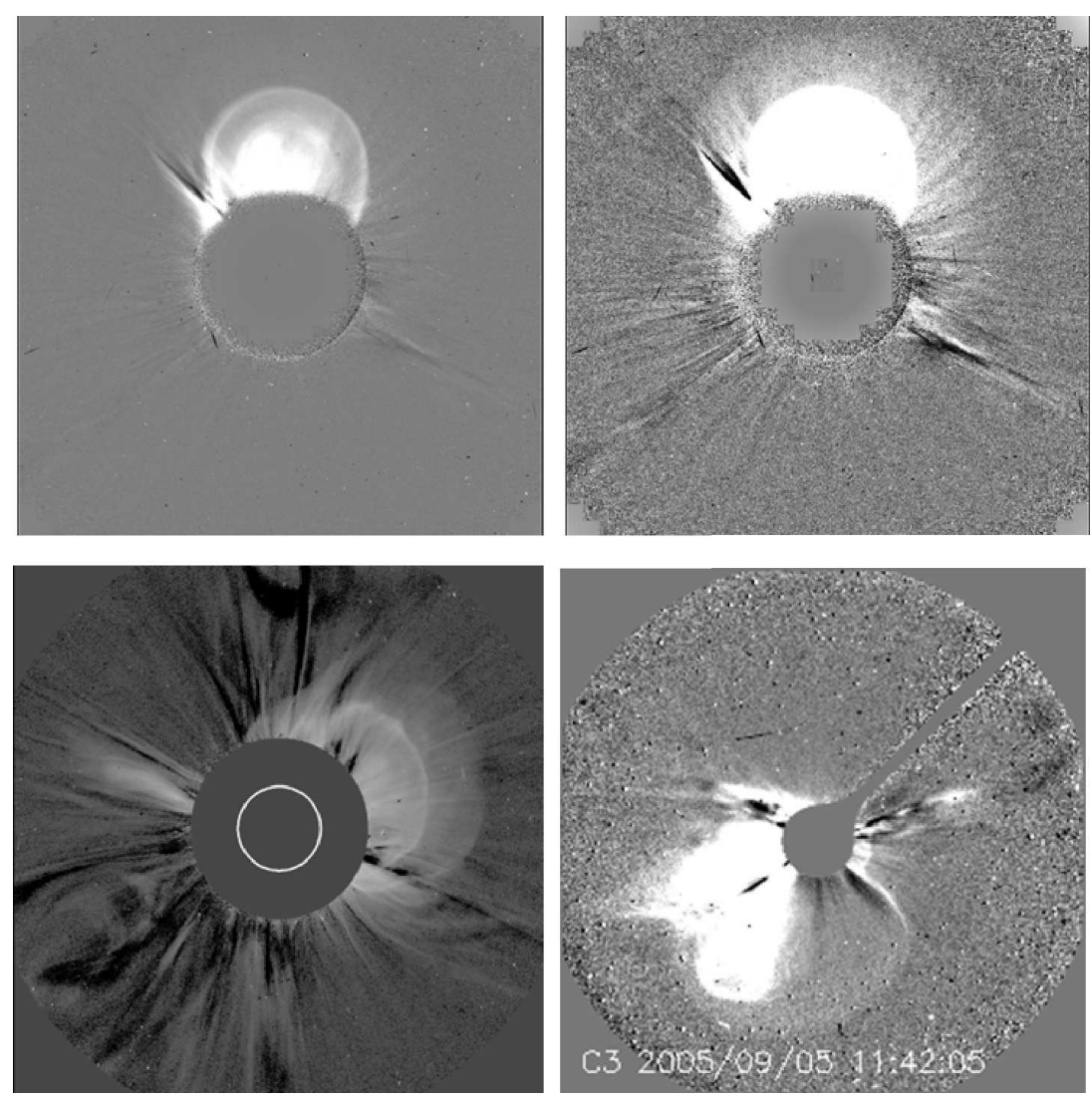

Figure 3. Upper left: An excess mass image of a $\mathrm{CME}$ in the $\mathrm{LASCO} / \mathrm{C} 2$ field of view. The event can be easily classified as a 3-part CME. A deflected streamer is located to the east without an apparent connection to the CME. Upper right: The same image displayed with a higher contrast ratio. A faint arch front terminating at the eastern deflected streamer is now visible. Indications of several other deflected streamers (or sub-streamer structures) can be seen. Bottom panels: Other examples of shock fronts ahead of CMEs in C2 (left) and C3 (right).

(or shock) propagates through and hence is much fainter (see Ontiveros and Vourlidas (2009) for density profiles of these structures). Our examination of thousands of CME images (Section 6) reveals that the "faint front followed by a bright loop" is a common occurence and it can constitute a reliable signature for the identfication of both the shock and FR fronts in the images. An important benefit from this identification is a better interpretation of the structures in images of halo CMEs.

\subsection{Halo CMEs}

There is no physical reason to expect that halo CMEs are a differenct class of CMEs. 'Loop' and '3-part'-CMEs should occur as halos and their FR should appear on the images...somewhere. The common approach has been to identify the outer envelope of the halo with the FR and proceed to fit it with a circular 

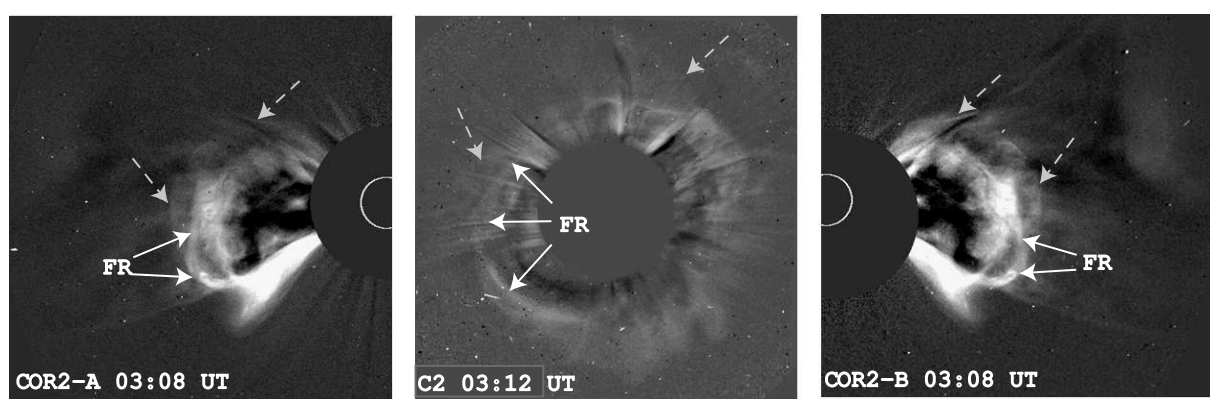

Figure 4. A demonstration of the appearance of 'two-front' morphology in a weak halo CME as viewed from LASCO/C2 (center). The CME appears as a 'loop'-CME in the SECCHI COR2-A (left) and B (right) coronagraphs. All three images are taken nearly simultaneously. The pile-up at the FR edge is marked by solid white arrows. The edge of the much fainter wave is marked by the dashed grey arrows. The existence of a shock is likely since the CME is propagating at $\sim 750 \mathrm{~km} \mathrm{~s}^{-1}$ in the LASCO field. The event occurred on February, 15, 2011 in association with an X-class flare. For similar examples see Vourlidas and Ontiveros (2009), Vourlidas and Bemporad (2012).

or elliptical cone models to extract kinematic parameters (Howard et al., 1982; Zhao, Plunkett, and Liu, 2002; Xie, Ofman, and Lawrence, 2004; Xue, Wang, and Dou, 2005). However, this approach is inconsistent with our theoretical understanding of FRs as more or less cylindrical structures, elongated along their axis. It is also inconsistent with the analyses of the CME projection effects (Cremades and Bothmer, 2004, Thernisien, Vourlidas, and Howard, 2009). Our discussion above solves this problem. To identify the FR in a halo CME image, we have to look for evidence of the 'two-front' morphology or of the bright loop structure alone when the CME is not fast enough to expect significant pileup at its wave front. Indeed, these structures are visible in the majority (if not all) of halo CMEs.

We picked a recent halo CME as an example (Figure 4). The event, which occurred on February 15, 2011, was the most symmetrical halo of the current solar cycle. It was associated with a large X-class solar flare, metric and decimetric Type-II emissions, and an EUV wave. We chose an event with relatively weak halo emission to demonstrate the robusteness of the feature detection. Much clearer examples are presented in Vourlidas and Bemporad (2012). See also Vourlidas and Ontiveros (2009) and Figure 7 (bottom left) for a single LASCO view. Returning to Figure 4 , we see that the halo CME appears as a regular 'loop', or even '3-part'-CME in the COR2-A and B fields of view. Note that all three images were taken nearly simultaneously and are differenced from a pre-event image. The FR boundary is readily identified in the COR2 images as a bright loop structure (marked by the white arrows and the label 'FR'). The same structure appears as a (fainter) loop in the C2 image. A still fainter front can be seen ahead of the loop. A wave compression, and possibly a shock, is expected in this event given its LASCO speed of $\sim 750 \mathrm{~km} \mathrm{~s}^{-1}$ and the Type-II radio emission. The wave is more difficult to discern in the side views from COR2 (dashed gray arrows) because the CME is projected against, and propagating though, a background corona disturbed by an earlier event. Even 
the streamer deflection and the wave associated with it can be detected, albeit barely, in the north (topmost gray arrow). The rugged set of features along the southern-southeastern part of the halo originate in the bright southern streamer, seen in the COR2 images.

This exercise shows that, with a little effort, we can identify the origins of the various features in a halo CME image and delineate the boundaries of the ejected FR with some precision. The important point here is that we do not have to rely on simplistic, rough approximations for the envelop of the CME. These would lead to imprecise measurements of the CME speed, size, and orientation with correponding implications for Space Weather predictions.

\section{Theoretical Support: Synthetic Images from MHD Simulations}

Thus far, we have only used coronagraph images to support our interpretation of CMEs as 3-part (5-part when a wave front appears) structures resulting from the expulsion of a magnetic FR from the Sun. We now turn to a numerical MHD simulation to determine which aspects of the coronal signatures identified in the LASCO images can be produced by an erupting three-dimensional FR. We analyze results obtained with an Adaptively Refined MHD Solver (ARMS; DeVore and Antiochos, 2008) simulation of the 'magnetic breakout' CME initiation mechanism (Antiochos, DeVore, and Klimchuk, 1999, Lynch et al., 2004, 2008 ) and the subsequent FR-CME propagation in the low corona to construct synthetic coronagraph images we can directly compare to observations.

\subsection{Description of the MHD Simulations and Eruption Overview}

The ARMS simulation data analyzed herein comes from the 'Left-Handed' CME eruption described by Lynch et al. (2009) in a fully 3-dimensional, globally multipolar magnetic field configuration. The solar atmosphere is initally in gravitationally stratified equilibrium with spherically symmetric density, pressure, and temperature profiles given in Lynch et al. (2008). The maximum field strengths in the $\mathrm{AR}$ are $\pm 40 \mathrm{G}$ which, while lower than observed values by anywhere from 10-100, yield a low- $\beta$ plasma in the CME source region $\left(\beta \sim 10^{-3}\right)$ and throughout the computational domain. Thus, the ARMS simulation data provide a physically valid, albeit idealized, representation of the magnetically-driven eruption process. Here, we briefly review the phases of the moderate speed Lynch et al. (2009) breakout CME eruption:

1. Energization $(0 \leq t \leq 10000 \mathrm{~s})$ : Surface shearing flows are applied adjacent to the polarity inversion line (PIL) of the active region (AR) resulting in the gradual accumulation of magnetic energy $\left(E_{\mathrm{M}} \sim 10^{31} \mathrm{ergs}\right)$ as the low-lying, strong AR fields are stressed. This sheared field component parallel to the AR PIL will become the FR-CME axial field.

2. Breakout Reconnection $(t \gtrsim 5000 \mathrm{~s})$ : As the sheared portion of the AR flux expands, the overlying coronal null point becomes distorted and flattened, forming a current sheet at the separatrix between the AR and background flux 
system. Continued expansion compresses the current sheet and drives magnetic reconnection which transfers overlying restraining background flux out of the way of the expanding stressed field which, in turn, increases expansion, and drives more reconnection in a runaway positive feedback scenario. The breakout reconnection facilitated expansion shows up as a smooth increase in kinetic energy to $E_{\mathrm{K}} \sim 3 \times 10^{29}$ ergs.

3. Eruptive Flare Reconnection $(t \gtrsim 10000 \mathrm{~s}$ ): The runaway sheared arcade expansion drive the formation and elongation of a radial current sheet above the PIL leading to the start of flare reconnection in the shear channel. The eruptive flare reconnection rapidly releases stored magnetic energy $\left(\Delta E_{\mathrm{M}} \sim\right.$ $7 \times 10^{30} \mathrm{ergs}$ ) through the magnetic reconfiguration and formation of flare loop arcades, supplies material and momentum to the ejecta via strong reconnection jet outflow $\left(E_{\mathrm{K}}\right.$ increases to $1.05 \times 10^{30} \mathrm{ergs}$ ), and, in the breakout model, creates the magnetic flux rope during the eruption process by generating highly twisted flux surrounding the erupting sheared field core.

The top row of Figure 5 plots representative magnetic fieldlines for $t=\{11000$, $12000,13000\}$ seconds during the CME eruption. Fieldlines representing the FR sheared field core are plotted in green, the reconnection-created FR CME twist component in magenta, and the background field in dark blue.

There are two primary challenges associated with MHD modeling of very fast CMEs and their subsequent shock generation in the low corona. The first is a correct description of the thermodynamics, field and plasma structure of the steady-state background solar wind. The lack of a single, widely-accepted theory for coronal heating means, in practice, every simulation relies on idealized, parametrized heating terms and calculates the resulting solar wind and open field structure from the balance of forces. The second major modeling challenge is overcoming the computational limitations associated with the magnetic field strength in CME source regions. The MHD numerical timestep is limited by the Alfven speed which makes the temporal evolution of kilogauss fields that are routinely observed in large active regions prohibitively expensive. Despite these model limitations, numerical MHD simulations are becoming increasingly sophisticated and capable. For example, the field strengths and self-consistent preeruption energization used by Lynch et al. (2008) and Roussev, Lugaz, and Sokolov (2008) were sufficient to initiate CMEs with eruption speeds on the order of 1200-1400 km/s. In simulations that bypass the difficulty of the pre-eruption evolution, fast eruptions can be generated with CME speeds $>2000 \mathrm{~km} / \mathrm{s}$, drive shock formation as low as $\sim 1.6 R_{\odot}$, and can produce complex white-light structures in synthetic coronagraph and HI images (Roussev, Lugaz, and Sokolov, 2008; Manchester et al., 2008; Lugaz et al., 2011).

\subsection{Comparison Between Synthetic and LASCO CME Images}

To construct synthetic coronagraph images, we use a version of the SolarSoft routine eltheory pro to calculate the total brightness in a regular 2D Cartesean 

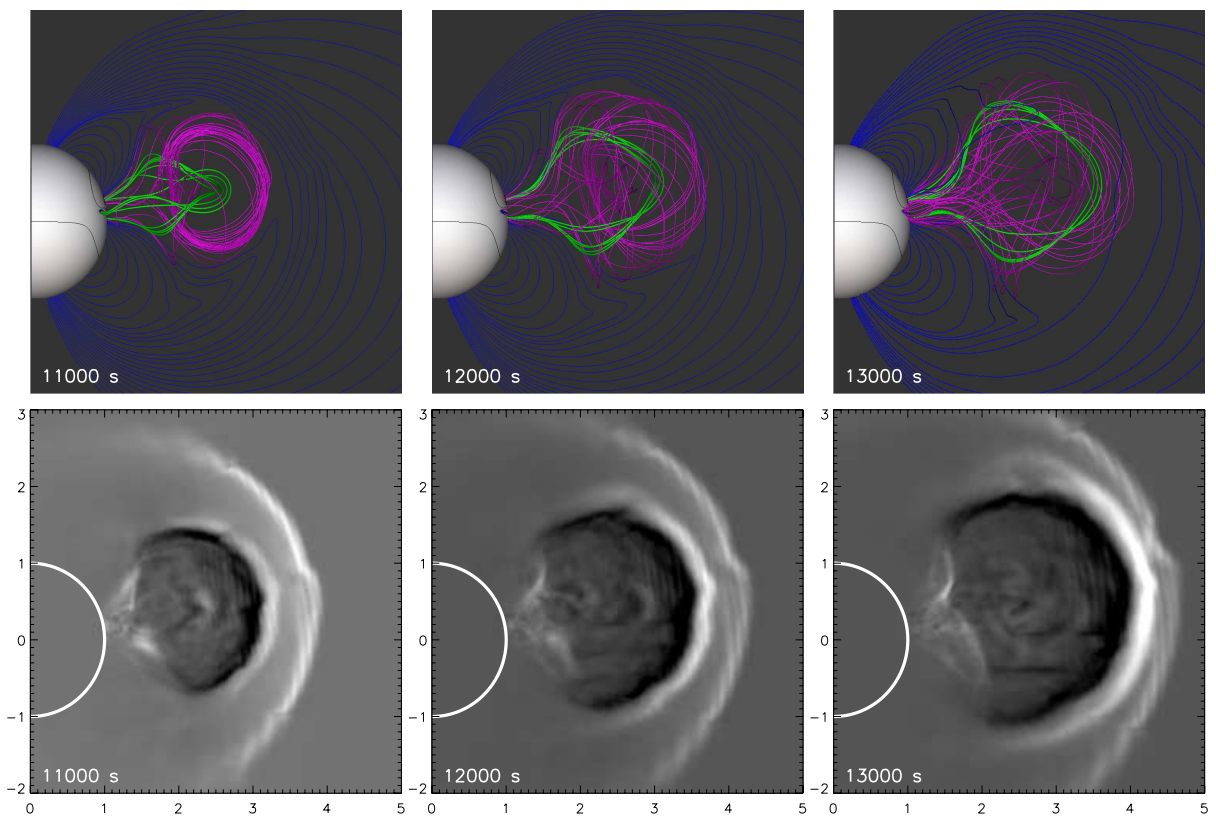

Figure 5. Top row: MHD simulation results of a breakout CME eruption in the coronagraph field of view for a limb event. The FR ejecta fieldlines are green, magenta for the sheared field core and reconnection generated twist flux. Bottom row: Synthetic running-difference (RD) coronagraph images constructed from the simulation's density evolution.
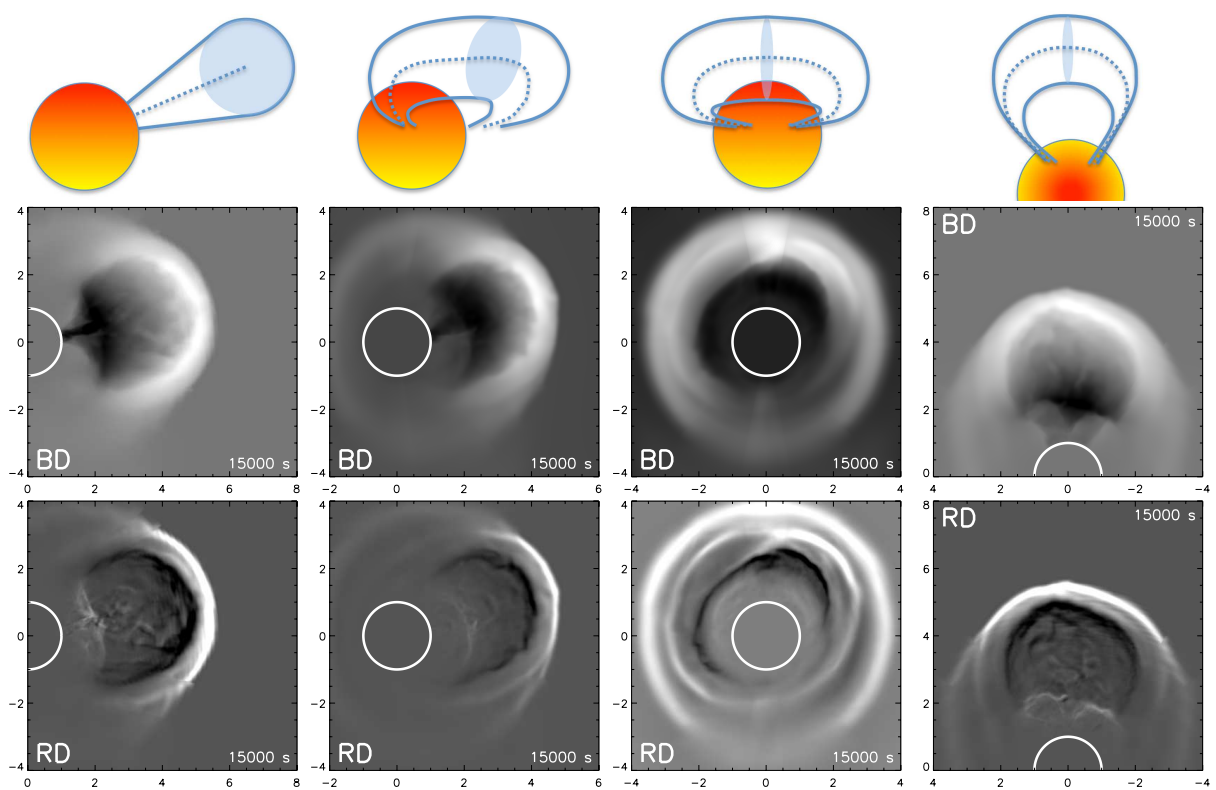

Figure 6. Top row: Schematic images depicting the 3D FR orientation with respect to the synthetic image POS. The middle, bottom rows plot the synthetic base-difference (BD), running-difference $(\mathrm{RD})$ images, respectively. Both the bright CME front and the CME-driven shock/expansion wave can be seen in these images. 
array of lines of sight that sample the spherical 3D MHD density data at every simulation output time. From these synthetic total brightness images we then construct base-difference (BD) images as $B(t)-B(0)$ and running-difference (RD) images as $B(t)-B(t-\Delta t)$. The temporal cadence, $\Delta t$, of our simulated data is $250 \mathrm{~s}$. The bottom row of Figure 5 plots the synthetic RD images such that the radial propagation of the center of the FR CME lies exactly in the RD image plane of the sky (POS) corresponding to the viewpoint of the $3 \mathrm{D}$ fieldline visualization.

In Figure 6 we have constructed a series of viewpoint orientations to examine morphological features of the synthetic coronagraph images. The top row indicates schematically the CME orientation with respect to the image POS and the middle, bottom rows plot the corresponding $\mathrm{BD}$, $\mathrm{RD}$ images respectively. From left to right, the angles between the radial propagation of the center of the FR CME and the image POS are $\{0,45,90,0\}$ degrees, with the fourth column representing a vantage point from the North solar pole looking down on the eruption.

The shock front, the bright CME front, and the FR cavity are each clearly seen in either the RD or BD images. The CME front morphology varies between a loop-like CME (within $45^{\circ}$ from the limb) to a halo $\mathrm{CME}$ (at $90^{\circ}$ from the limb) similarly to the actual observations.

While the idealized MHD simulation produces both the shock front/expansion wave and the bright leading edge of the ejecta ahead of the FR CME driver, there is no corresponding high density plug of material associated with the FR core. This limitation was also present in the axisymmetric models (e.g., Lynch et al., 2004 and is due largely to our simplified pre-eruption coronal density distribution which does not include prominence material along the low-lying sheared field or the enhanced densities associated with either ARs or that would arise in a closed-field streamer belt geometry. Our simplified model background results in two main consequences. First, without dense material tracing the topology of the FR CME core, it is difficult to distinguish between the edge-on view (Figure 6 first column, looking at the FR cross-section; compare to Figure 1) and the topdown view (Figure 6 fourth column, FR axis lies in the image POS; compare to Figure 3. bottom left) in the synthetic images. Second, the relative brightness of the CME leading edge and the shock/expansion wave do not have the same ratio as commonly observed in the coronagraph images (which, as discussed earlier, require significant contrast enhancement). Furthermore, the lack of a background with coronal streamer structures in various locations does not allow us to compare streamer deflections or the effects of coronal hole locations in this particular simulation, although these issues are an area of active research (see, e.g., Lugaz et al., 2011; Zuccarello et al., 2012).

We also note that the synthetic shock appears very close to the CME leading edge (the driver) which is not the case for the LASCO images we have presented so far. This is an evolutionary effect, however, as Figure 5 has already shown. In our simulations, the shock is initially clearly ahead of the CME which catches up to it within $\sim 2000 \mathrm{sec}$. Of course, different speed profiles and ambient coronal configurations will result in different standoff distances. Again, our comparison 

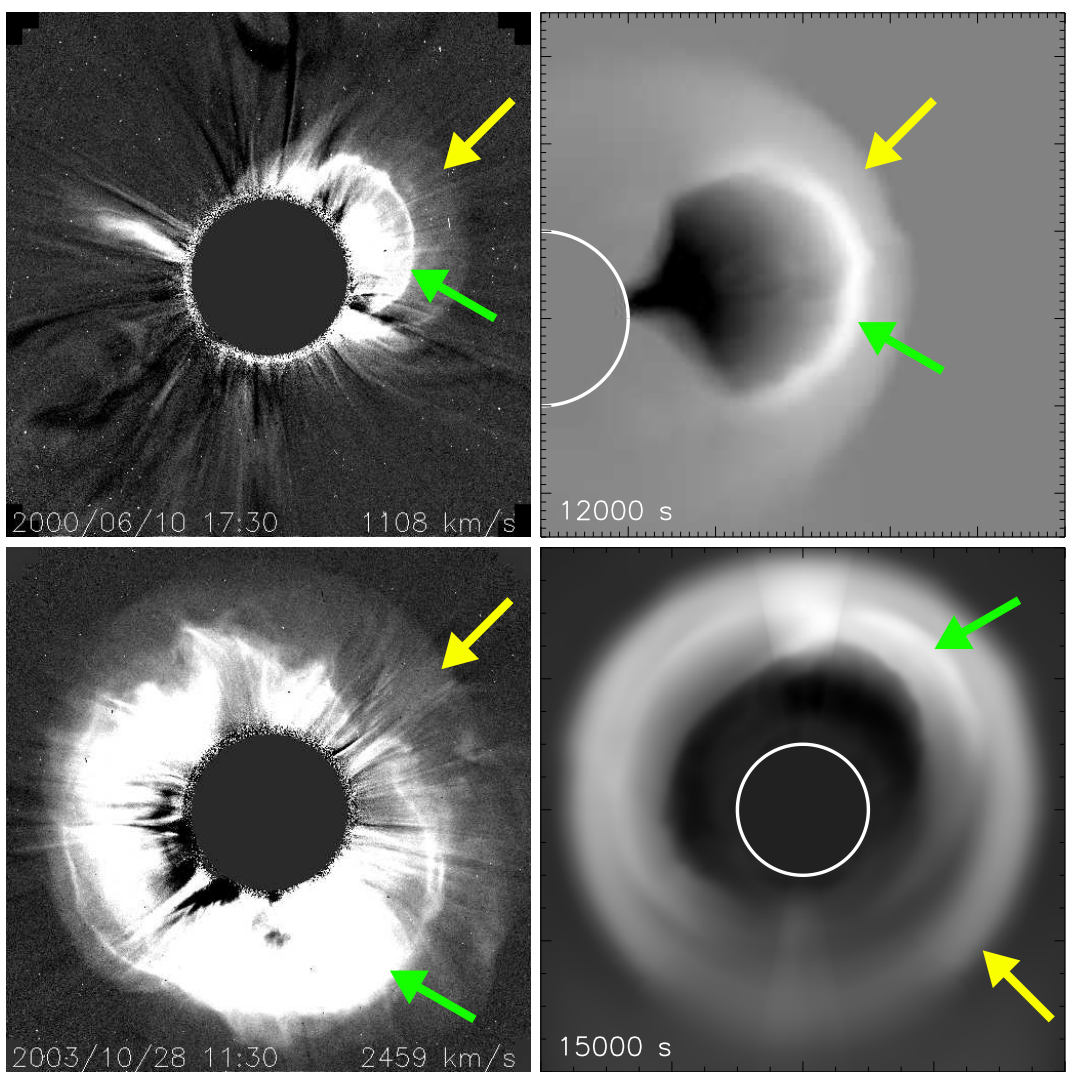

Figure 7. Comparison between LASCO excess mass images (left column) and synthetic base-difference images from our MHD simulations (right column). There is a very good correspondance between observed and simulated structures, despite the idealized nature of the simulation. The yellow arrows denote the shock/expansion wave front and the green arrows denote the CME leading edge density enhancement. The projected speeds for the LASCO CMEs are also shown.

here is not an attempt to model a specific CME event with a realistic background density distribution, but rather to present an idealized general case of the appearance of a generic shock-driving FR-CME in a coronagraph field of view.

It is precisely the generality of our simulation that makes the comparison to two LASCO events especially striking, as illustrated in Figure 7. The simulated synthetic BD images (right column) show the diffuse intensity region leading the bright CME front which is associated with the boundary of the magnetic FR structure exactly as we proposed in Sections 2 and 3 . Yellow arrows denote the shock front, green arrows denote the CME leading edge. Despite not capturing the observed sharpness or intensity of the CME front, the simulations do show that the faint halo outline in the LASCO images corresponds to the shock envelope. Therefore, our 5-part CME structure is a completely straight-forward interpretation and a natural consequence of the eruption of a 3D FR CME. 


\section{The Relationship between Ejected Prominence and CME Flux Rope}

Having discussed the nature of the front and the cavity, we turn our attention to the core, the last component of '3-part'-CMEs. The core has been associated with the erupting prominence ever since combined $\mathrm{H} \alpha$ and coronagraphic observations demonstrated the colocation of the two structures (e.g., Hildner et al., Illing and Hundhausen, 1975, 1985). The fuzziness of the core compared to the filamentary structure of prominences in $\mathrm{H} \alpha$ and $\mathrm{He}$ I observations is attributed to the progressive ionization and heating of the chromosheric plasma within the prominence as the structure erupted outwards.

Of the many unclear aspects on the role and evolution of prominence in eruptive events, there are two that pertain to our discussion here. Namely, what is the spatial relationship between the core (and prominence) to the cavity and, why in-situ detections of chromospheric material within CMEs are so rare.

The first question stems from the early interpretations of the prominence as a flux rope and its identification with the CME cavity (Low and Hundhausen, 1995). Although prominences exhibit helical structures when they erupt, the large number of combined observations with LASCO (in white light) and EIT (in He I $304 \AA$ ) have shown that the cavity is not the prominence itself. They have also shown that the core does not lie in the center of the cavity as was thought in the past (see Figure 10 in Cliver et al. 1986). Rather the prominence lies at the bottom of the cavity, the cool plasma suspended in the dips of the FR field lines by the balance between gravity and magnetic tension forces.

While these concepts are widely accepted thanks to the extensive observations of quiescent cavities and prominences (Gibson et al., 2006), there seems to be a lingering confusion on the location and importance of the prominence relative to the erupting FR or cavity. The high resolution observations from SECCHI and Atmospheric Imaging Assembly (AIA; Lemen et al., 2012) can now put this issue to rest. For example, Régnier, Walsh, and Alexander (2011) have presented multi-wavelength AIA observations of a polar crown filament during its early eruption stages where they image both the cool prominece and the hotter FR. The high spatial resolution of AIA reveals cool prominence plasma in $304 \AA$ embedded in field lines at the bottom of the FR visible in $193 \AA(\sim 1.4 \mathrm{MK})$. The $193 \AA$ emission has the typical 'horn' morphology we discussed in Section 2 and frequently seen in the EIT images during Cycle 23. To demonstrate how common are these structures, we present two more examples in Figure 8.

On the left, we show a snapshot from a prominence eruption at the northeast limb on June 12, 2010 captured by the AIA instrument. To demonstrate the relative locations of the FR and prominence we combined the $193 \AA$ (gold) and $304 \AA$ (red) AIA channels after enhancing the individual images through our wavelet processing algorithm (Stenborg, Vourlidas, and Howard, 2008). The accompanying movie demonstrates two important aspects of the eruption: (1) the pre-existing cavity is not actually empty but it is filled with plasma at coronal temperatures, and (2) most of the cool prominence material returns to the surface and is not ejected with the rest of the CME.

On the right, we show a similar observation from SECCHI/EUVI-A taken on February 28, 2010. Here the $195 \AA$ images are shown in silver color. We observe 

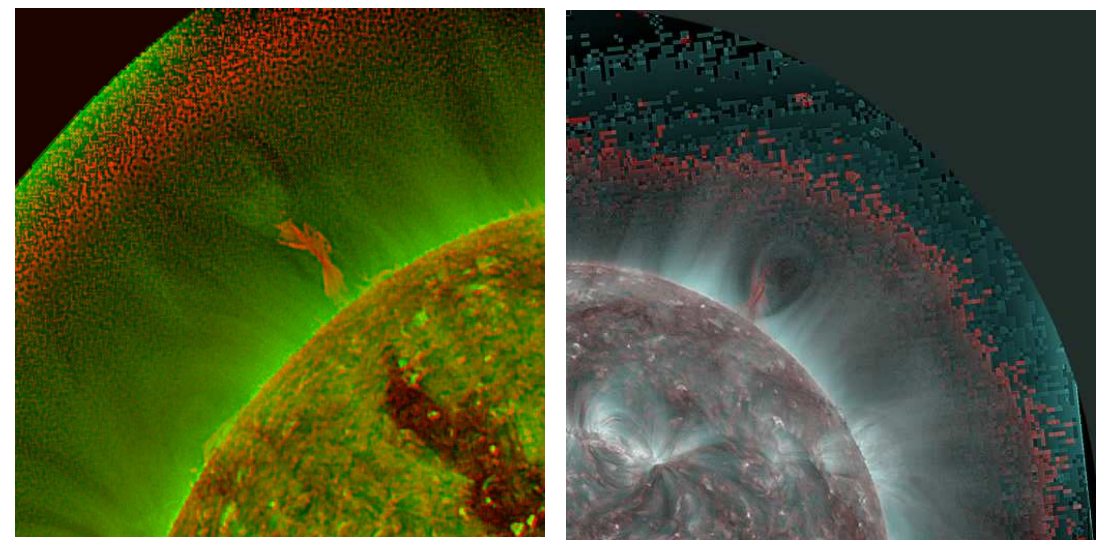

Figure 8. Left: Image of a prominence eruption at the northeast limb on June 12, 2010 using a composite of AIA $193 \AA$ (green) and $304 \AA$ (red) channels. Right: Snapshot of a prominence eruption at the northwest limb on February 28, 2010 using a composite of EUVI-A $195 \AA$ (silver) and $304 \AA$ (red) channels. Both images are snapshots from the online movies.

strong circular motions in the center of the cavity during the early part of the event previously noted by Wang and Stenborg (2010) who did not comment on the temperature characteristics on these motions. Here, we can see that the motions are associated with extensions of $304 \AA$ emission into the center of the cavity which exhibits a very clear FR morphology. The cool plasma seems to disappear after its injection until a new extension brings in another quantity of cool material into the cavity. Hence, the rotations within the cavity seem to be driven by the episodes of heating of chromospheric plasma. In contrast to the June 12, 2010 event, the prominence erupts carrying a significant amount of cool plasma outwards. No return flows are evident in this event.

These EUV observations are quite clear. The prominence is not the cavity. It is the core. The core is not located at the center of the cavity. It is located at the trailing edge. It may, however, appear to be at the center or at other locations due to projection effects. Much of the chromospheric material either drains back to the surface or heats to coronal temperatures or both during the early stages of the eruption. This is likely the reason for the scarcity of in-situ detections of such cool material in the inner heliosphere.

Finally, one may question the generality of our conclusions since we have used as examples prominence eruptions from polar crown and generally quiescent areas of the corona. This was done mainly for convenience. Polar crown filament eruptions are both spectacular and slow thus providing a large sample of clear highly detailed structures at various stages of activity. It is much harder to find fast, explosive events with clear structures due to their fast evolution and the large disturbances they create in the surrounding corona. However, detailed analyses of impulsive events do reach the same conclusions. For example, Patsourakos, Vourlidas, and Stenborg (2010) were able to detect the expanding cavity and follow the formation of the 3-part structure within the center of an active region during a very impulsive event (peak acceleration $\sim 1.5 \mathrm{~km} \mathrm{~s}^{-2}$ ). Similarly, Cheng et al. (2012) obtained clear distance-time plots for an expanding 
cavity followed by a spectacular filament for an event reaching (short-lived) accelerations close to $3 \mathrm{~km} \mathrm{~s}^{-2}$.

\section{Revisting the LASCO Statistics: How Many FR-CMEs Are There?}

Having addressed the origins of the various sub-structures of a CME in the coronagraph and EUV images,we are now in position to answer one of the most common questions on CME studies: How many FR-CMEs are there? As noted in the introduction, the last studies to undertake that question used Solar Maximum Mission (SMM) data (Burkepile and St. Cyr, 1993 Krall, 2007). The answer $(\sim 30 \%)$ has been quoted ever since. But does it still hold after the observations of thousands of CMEs? Besides, we want to make sure that our examples of FR-indicative CME morphologies are indeed representative of the CME phenomenon as a whole?

\subsection{Morphological Classfication of the CDAW Database}

So we have undertaken the task to go over the full CDAW database and visually classify the events according to their moprhology. Based on our discussion so far, and on our personal experience with the LASCO images, we decided to classify the events into five categories (compared to the ten categories in Howard et al. 1985) as follows:

- Flux Rope: CMEs that exhibit a clear 3-part morphology (Figure 1).

- Loop: CMEs with a bright,filamentary loop but otherwise lacking a cavity and/or a core. Good indicators for the existence of shock ('two-front' morphology, Section 3 )

- Jet: Narrow CMEs $\left(\lesssim 40^{\circ}\right)$ lacking a sharp front, detailed sub-structure, or circular morphology.

- Failed: Events that disappear in the C3 field of view despite being bright enough in the C2 field to be labelled as 'CMEs'. Their disappearance cannot be explained by lack of observations, overlapping CMEs, or other instrumental reasons. These events were discussed in Vourlidas et al. (2010).

- Outflow: Events wider that jets, without clear loop front or cavity. They can as large as regular CMEs and can contain filamentary material (Figure 9 . left).

- Unknown: This 'catch-all' category contains mostly wrongly identified events, events with too few observations $(<4)$, and events that cannot be classified in any of the other categories due to poor observations, such as presence of cosmic rays or data dropouts (Figure 9 right).

First, we excluded events with width less than $20^{\circ}$ and with less than four observations. To do the classification, we used only mass images (Vourlidas et al., 2010). We displayed all available mass images (LASCO C2 and C3) for each event with the same contrast $\left( \pm 5 \times 10^{10} \mathrm{gr} / \mathrm{pix}\right)$. When the morphology was 

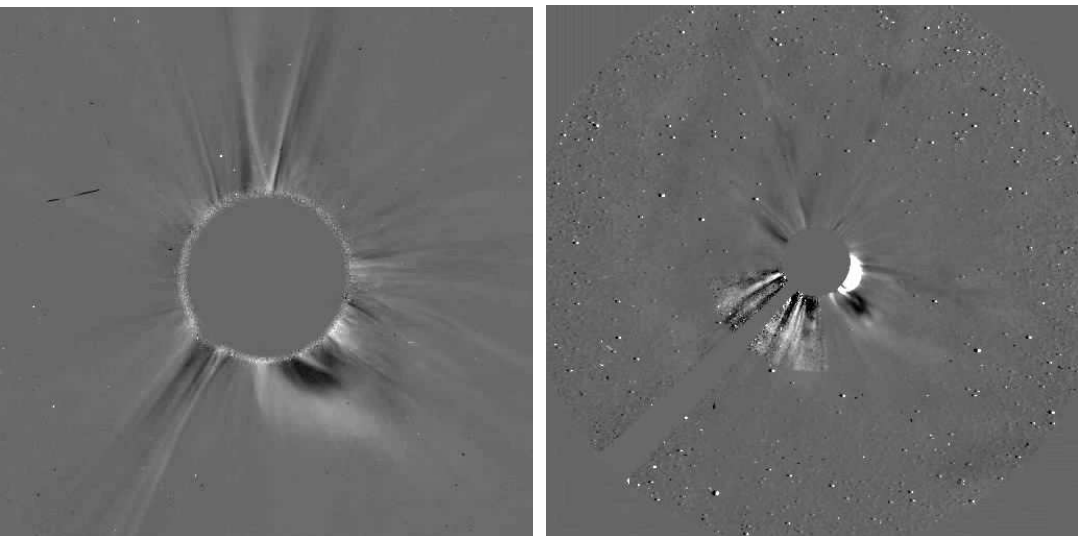

Figure 9. Left: Example of an 'Outflow' event. The CME lacks a 3-part morphology, it is too wide to be a 'Jet' event, and the front is not sharp enough to be classified as a 'Loop' CME. Right: Example of an 'Unknown' category event. The event propagating along the C3 occulter does not show any of the characteristics of a CME and it is too faint to provide accurate measurements for any parameter. Such events should not be included in CME lists. Both images are snapshots from the online movies.

Table 1. Time history of the CME eruption as marked by several key events.

\begin{tabular}{lrrrrrrr} 
Type & 1997 & 1998 & 2000 & 2002 & 2004 & 2008 & 2010 \\
\hline Flux Rope & 29 & 42 & 86 & 54 & 53 & 35 & 154 \\
Loop & 31 & 43 & 191 & 100 & 79 & 9 & 56 \\
Outflow & 48 & 96 & 282 & 145 & 126 & 56 & 209 \\
Jet & 5 & 16 & 85 & 17 & 32 & 5 & 32 \\
Failed & 10 & 33 & 54 & 10 & 67 & 14 & 30 \\
Unknown & 74 & 98 & 276 & 161 & 220 & 42 & 113 \\
\hline Total & 224 & 357 & 984 & 597 & 587 & 162 & 476 \\
FR-CMEs & $40 \%$ & $33 \%$ & $39 \%$ & $35 \%$ & $36 \%$ & $37 \%$ & $43 \%$ \\
\hline
\end{tabular}

not apparent, we used a movie of the mass images to get a better sense for the development of the event as a function of time and distance. We excluded the events in the 'Unknown' category from the statistics since we consider them as erroneous and/or unreliable detections. Our results are shown in Table 1.

\subsection{Statistical Results}

For the purposes of this work, we analyzed six out of the sixteen years of available CDAW mass measurements spread over Cycle 23. This is a sufficiently large sample to extract robust estimates for the rates of occurence of the various morphologies. We plan to report on the statistics of the full CDAW catalog in a forthcoming publication.

One of the first interesting results from this exercise is the rather large percentage of 'Unknown' events. They comprise 984 out of 3387 events or $29 \%$ of the 
sample. Recall that the 'Unknown' category includes events that are not CMEs or even outflows, events that are part of a larger CME and should not count as separate entries, and events with measurements ending prematurely. In other words, these events should not be counted in the statistics or other analyses of the CDAW catalog.

Excluding the 'Unknown' events, we find that the class of unambiguous FRCMEs, which consists of the sum of 'Flux Rope' plus 'Loop' classes, comprises $40 \%$ of the total number of CMEs (962/2403 events). There is no obvious correlation with solar cycle but there is a slight hint. The highest percentages of FR-CMEs occur in 1997 and 2010. It remains to be seen whether this result is statistically significant. We emphasize that the $40 \%$ of FR-CMEs is a lower limit for the existence of FRs. Some of the 'Outflow' events could be FRs. Indeed many contain hints of '3-part' structures or cavities, but they are either too faint or the background corona is too disturbed by previous events, to make a conclusive classification. So we choose to err on the side of caution and not include them in the FR-related classes, at this point.

\section{Discussion and Conclusions}

Our aim is to provide convincing evidence of the CME as an erupting FR. To that end, we have used a variety of EUV and white light observations, MHD simulations, statistics, and have considered projection effects and theoretical predictions. Leaving the question of CME initiation aside, we found that the following picture can lead to a self-consistent interpretation of the observations across many wavelength ranges and is in agreement with the majority (if not all) of our current theoretical understanding of explosive energy release from the Sun.

Basically, a CME is the eruption of a magnetic flux rope with its emission measure dominated by coronal temperature plasma, carrying a prominence along its bottom dips, piling up the overlying streamer plasma, and driving a wave ahead (if the acceleration is sufficently high). This interpretation has long been adopted for the '3-part'-CMEs, as we discussed earlier.

The novelty in this work is the interpretation of the bright loop front as the pileup of material at the boundary of the flux rope irrespective of the ' 3 -part' appearance. The interpretation is supported strongly by the MHD simulations and straightforward physical reasoning (Section 4). A FR structure propagating through plasma presents an extended obstacle against which the material is piled up and transported outwards. The narrow width and brightness of that front further suggests that the pileup occurs over a sharp boundary. Such a boundary is expected between the closed FR fields and the ambient magnetic field. The sharpness of the boundary may depend on the rate of magnetic field influx in the FR during its formation or the initial acceleration and starting height. Such effects have important connections to theories of CME initiation and can be investigated now.

The other novelty is the introduction of the 'two-front' morphology by pointing out the existence of faint, relatively sharp, fronts ahead of the bright loop 
front. The interpretation of the faint front as density compression by a wave (or shock, depending on speed) is again supported by MHD simulations, observations and physical expectations. The stark observational differences between the bright sharp front and diffuse front clearly point to a different origin. The diffuse fronts are: well-defined, faint, followed by diffuse emission, can be very extended, and envelope the sharp fronts. The sharp fronts, in turn, are: sharp, bright, followed by emission depletions, have well-defined extents, and are behind the diffuse fronts. The faint fronts appear only during fast eruptions and their characteristics, especially the weakness of their emission and lack of post-front depletion are strong indications that these fronts are results of local density compression and not of transported piled-up plasma. The, albeit few, 3D reconstructions of the density profile across the front (Ontiveros and Vourlidas, 2009) can readily explaining the profile as a result of LOS integration and recover compression ratios in agreement with theoretical expectations (less than 4). Besides its importance for understanding coronal shocks, the identification of the 'two-front' morphology allows an understanding of the geometry of halo CMEs as it can help us distinguish among shock, streamer deflections and FR signatures in the coronagraph images. In that way, we can now obtain accurate outlines of the FR (or the shock, depending on the problem at hand) which should lead to better inputs to CME propagation models.

The identification of these two features leads to a much simpler classification of CME white light morphologies. We used four categories (ignoring the 'Unknown' category) compared to nine in Howard et al. (1985). Two of them ('FR' and 'Loop') refer to the same FR instrinsic structure as we have argued. JetCMEs also contain helical structures as recent research has shown (Patsourakos et al., 2008: Pariat, Antiochos, and DeVore, 2009, Nisticò et al., 2010). Thus, our classification is essentially reduced to events with and events without apparent helical topologies. The helical topology may not be visible in the latter for several reasons. They may propagate at large angles from the POS (Sheeley and Wang, 2007) or through areas disturbed by previous events. They may be too compact to discern their cavity morphology without favorable projections (Wang and Sheeley, 2006). Finally some of these events do not appear to be CMEs in the first place failing to reach large distances in the corona (called 'failed' CMEs by Vourlidas et al. , 2010). A certain number of the remaining events appear to be related to $\mathrm{H} \alpha$ and/or $304 \AA$ surges similar to the event studied by Vourlidas et al. (2003). The low coronal signatures of these events do not exhibit any particular morphology or geometry and hence tend to appear as semi-amorphous clouds, with the occassional traces of cool material.

Our final estimate of $41 \%$ for the rate of occurrence of FR-CMEs in the LASCO data may not look very differernt from the widely quoted number of $30 \%$. However, one must first consider the size of the event samples in past morphological works. Munro et al. (1979) reported a $26 \%$ occurence of 'Loop'CMEs in a sample of 77 SMM CMEs while Wagner (1984) found loop and bubble CMEs in $80 \%$ of 65 SMM CMEs. Obviously, selection bias is important with such small event samples. The largest morphological study to date categorized 998 Solwind CMEs of which $31.3 \%$ belonged to an FR-CME class (we summed the statistics for the following structural classes, curved front, loop, streamer 
blowout, fan) (Howard et al., 1985). We base our statistics here on a sample of 2970 events, $3 \times$ larger than the Solwind sample and is still expanding. We will classify the full LASCO database in the near future. Therefore, we feel that our numbers in Table 1 are quite robust and a large improvement over past work.

The central question of this Topical Issue is whether all CMEs are flux ropes. To provide a conclusive answer (to the extent possible in science), we attacked the problem in several ways: multiple viewpoint coronagraphic observations of CMEs, multi-thermal EUV observations of the pre-erupting structures, 3D MHD simulations, and large sample statistics. We summarize our findings as follows:

- The detection of a bright filamentary front in CMEs is a clear indication of the existence of a FR even if the event does not exhibit the classical 3-part morphology.

- At least $41 \%$ of CMEs exhibit clear FR signatures ('3-part or 'loop') in the coronagraph images.

- The 'two-front' morphology (faint front followed by a bright loop) is a reliable indicator of a CME-driven wave (or shock, depending on speed).

- The FR can be separated from the shock signatures in images of halo CMEs at least in locations where the bright loop appears.

- MHD simulations are able to capture the main structural properties of white light CMEs.

- The prominence is not the cavity and is not the FR but is the core. The cool prominence material rests on the dips of the field lines comprising the FR (in the case of pre-existing FR, at least).

- The majority of the prominence material either drains to the surface or is heated to coronal temperatures during the early phases of the eruption. This may be the reason for the scarcity of in-situ detections of cool material.

- A typical fast CME comprises five parts: shock front, diffuse sheath, bright front, cavity, and core.

Our discussion suggests that it is time to rethink the original definition for a CME (Hundhausen et al., 1984), as expressed in Schwenn, 2006. "We define a CME to be an observable change in coronal structure that 1) occurs on a time scale of a few minutes and several hours and 2) involves the appearance (and outward motion) of a new, discrete, bright, white light feature in the coronagraph field of view." This definition manages to be broad (no mention of the physical origin or nature of the 'structure') and narrow (CME is defined as a white light feature observed by a coronagraph) at the same time. It may have been an appropriate definition during the times of exploratory CME research, sparse wavelength coverage, and simplified physical models. But times have changed. We are regularly studying CMEs with multiple instruments and wavelengths, have accumulated CME observations spanning a full solar cycle, and are asking highly detailed questions with their modeling. Thus, it may be useful to derive a more precise CME definition using physically-based terms, at least for the events exhibiting clear FR structures (FR-CMEs). Based on the work presented here and in Vourlidas et al. (2010), we propose the following definition:

We define an FR-CME to be the eruption of a coherent magnetic, twistcarrying coronal structure with angular width of at least $40^{\circ}$ and able to reach beyond $10 R_{\odot}$ which occurs on a time scale of a few minutes to several hours. 
The next challenge now is whether we can apply this definition to all CMEs (hence replace 'FR-CME' with 'CME' above). In other words, we propose that the proper questions we should be asking is not 'are all CME flux ropes?' but rather 'Are there any CMEs that are not FR-CMEs?'

Acknowledgements The work of AV and RAH is supported by NASA contract S-136361$\mathrm{Y}$ to the Naval Research Laboratory. BJL and YL acknowledge support from AFOSR YIP FA9550-11-1-0048, NASA NNX11AJ65G, and NNX08AJ04G. We thank G. Stenborg for his continuing efforts to provide better quality solar images. SOHO is an international collaboration between NASA and ESA. LASCO was constructed by a consortium of institutions: the Naval Research Laboratory (Washington, DC, USA), the Max-Planck-Institut fur Aeronomie (Katlenburg-Lindau, Germany), the Laboratoire d'Astronomie Spatiale (Marseille, France) and the University of Birmingham (Birmingham, UK). The LASCO CME catalog is generated and maintained at the CDAW Data Center by NASA and The Catholic University of America in cooperation with the Naval Research Laboratory. The SECCHI data are produced by an international consortium of the NRL, LMSAL and NASA GSFC (USA), RAL and Univ. Bham (UK), MPS (Germany), CSL (Belgium), IOTA and IAS (France).

\section{References}

Antiochos, S.K., DeVore, C.R., Klimchuk, J.A.: 1999, Astrophys. J. 510, 485. doi $10.1086 / 306563$

Anzer, U., Pneuman, G.W.: 1982, Solar Phys. 79, 129. doi $10.1007 /$ BF00146978

Bemporad, A., Mancuso, S.: 2010, Astrophys. J. 720, 130. doi $10.1088 / 0004-637$ X/720/1/130

Brueckner, G.E., Howard, R.A., Koomen, M.J., Korendyke, C.M., Michels, D.J., Moses, J.D., Socker, D.G., Dere, K.P., Lamy, P.L., Llebaria, A., Bout, M.V., Schwenn, R., Simnett, G.M., Bedford, D.K., Eyles, C.J.: 1995, Solar Phys. 162, 357. doi 10.1007/BF00733434

Burkepile, J.T., St. Cyr, O.C.: 1993, Tech. Note TN-369+STR 93, 26556.

Burlaga, L.F., Klein, L., Sheeley, N.R. Jr., Michels, D.J., Howard, R.A., Koomen, M.J., Schwenn, R., Rosenbauer, H.: 1982, A magnetic cloud and a coronal mass ejection. 9, 1317. doi 10.1029/GL009i012p01317

Cane, H.V., Richardson, I.G.: 2003, Journal of Geophysical Research (Space Physics) 108, 1156. doi $10.1029 / 2002 J A 009817$

Chen, J., Garren, D.A.: 1993, Interplanetary magnetic clouds: Topology and driving mechanism. 20, 2319. doi 10.1029/93GL02426

Chen, J., Howard, R.A., Brueckner, G.E., Santoro, R., Krall, J., Paswaters, S.E., St. Cyr, O.C., Schwenn, R., Lamy, P., Simnett, G.M.: 1997, Astrophys. J. Lett. 490, L191. doi $10.1086 / 311029$

Chen, J., Santoro, R.A., Krall, J., Howard, R.A., Duffin, R., Moses, J.D., Brueckner, G.E., Darnell, J.A., Burkepile, J.T.: 2000, Astrophys. J. 533, 481. doi 10.1086/308646

Chen, P.F.: 2011, Living Reviews in Solar Physics 8, 1.

Cheng, X., Zhang, J., Olmedo, O., Vourlidas, A., Ding, M.D., Liu, Y.: 2012, Astrophys. J. Lett. 745, L5. doi 10.1088/2041-8205/745/1/L5

Cliver, E.W., Dennis, B.R., Kiplinger, A.L., Kane, S.R., Neidig, D.F., Sheeley, N.R. Jr., Koomen, M.J.: 1986, Astrophys. J. 305, 920. doi $10.1086 / 164306$

Cremades, H., Bothmer, V.: 2004, On the three-dimensional configuration of coronal mass ejections. 422, 307.

Crifo, F., Picat, J.P., Cailloux, M.: 1983, Coronal transients - Loop or bubble. 83, 143. doi $10.1007 /$ BF00148249.

Dasso, S., Nakwacki, M.S., Démoulin, P., Mandrini, C.H.: 2007, Solar Phys. 244, 115. doi $10.1007 /$ s11207-007-9034-2

Dere, K.P., Brueckner, G.E., Howard, R.A., Michels, D.J., Delaboudiniere, J.P.: 1999, Astrophys. J. 516, 465. doi 10.1086/307101

DeVore, C.R., Antiochos, S.K.: 2008, Astrophys. J. 680, 740. doi 10.1086/588011.

Forbes, T.G.: 1990, J. Geophys. Res. 95, 11919. doi 10.1029/JA095iA08p11919.

Gibson, S.E., Foster, D., Burkepile, J., de Toma, G., Stanger, A.: 2006, Astrophys. J. 641, 590. doi $10.1086 / 500446$ 
Gopalswamy, N., Thompson, W.T., Davila, J.M., Kaiser, M.L., Yashiro, S., Mäkelä, P., Michalek, G., Bougeret, J.-L., Howard, R.A.: 2009, Solar Phys. 259, 227. doi $10.1007 / \mathrm{s} 11207-009-9382-1$

Gosling, J.T., Hildner, E., MacQueen, R.M., Munro, R.H., Poland, A.I., Ross, C.L.: 1974, J. Geophys. Res. 79, 4581. doi 10.1029/JA079i031p04581.

Hildner, E., Gosling, J.T., Hansen, R.T., Bohlin, J.D.: 1975, Solar Phys. 45, 363. doi $10.1007 /$ BF00158456.

Howard, R.A., Michels, D.J., Sheeley, N.R. Jr., Koomen, M.J.: 1982, The observation of a coronal transient directed at earth. 263, L101. doi 10.1086/183932

Howard, R.A., Sheeley, N.R. Jr., Michels, D.J., Koomen, M.J.: 1985, Coronal mass ejections 1979-1981. 90, 8173.

Howard, R.A., Moses, J.D., Vourlidas, A., Newmark, J.S., Socker, D.G., Plunkett, S.P., Korendyke, C.M., Cook, J.W., Hurley, A., Davila, J.M., Thompson, W.T., St Cyr, O.C., Mentzell, E., Mehalick, K., Lemen, J.R., Wuelser, J.P., Duncan, D.W., Tarbell, T.D., Wolfson, C.J., Moore, A., Harrison, R.A., Waltham, N.R., Lang, J., Davis, C.J., Eyles, C.J., Mapson-Menard, H., Simnett, G.M., Halain, J.P., Defise, J.M., Mazy, E., Rochus, P., Mercier, R., Ravet, M.F., Delmotte, F., Auchere, F., Delaboudiniere, J.P., Bothmer, V., Deutsch, W., Wang, D., Rich, N., Cooper, S., Stephens, V., Maahs, G., Baugh, R., McMullin, D., Carter, T.: 2008, Space Science Reviews 136, 67. doi $10.1007 / \mathrm{s} 11214-008-$ 9341-4.

Hundhausen, A.J.: 1987, In: V. J. Pizzo, T. Holzer, \& D. G. Sime (ed.) Sixth International Solar Wind Conference, 181.

Hundhausen, A.J., Sawyer, C.B., House, L., Illing, R.M.E., Wagner, W.J.: 1984, J. Geophys. Res. 89, 2639. doi 10.1029/JA089iA05p02639

Illing, R.M.E., Hundhausen, A.J.: 1985, J. Geophys. Res. 90, 275. doi $10.1029 /$ JA090iA01p00275

Isavnin, A., Kilpua, E.K.J., Koskinen, H.E.J.: 2011, Solar Phys. 273, 205. doi 10.1007/s11207011-9845-z

Kaiser, M.L., Kucera, T.A., Davila, J.M., St. Cyr, O.C., Guhathakurta, M., Christian, E.: 2008, Space Science Reviews 136, 5. doi 10.1007/s11214-007-9277-0

Kim, R.-S., Gopalswamy, N., Moon, Y.-J., Cho, K.-S., Yashiro, S.: 2012, Astrophys. J. 746, 118. doi $10.1088 / 0004-637 X / 746 / 2 / 118$

Klein, L.W., Burlaga, L.F.: 1982, Interplanetary magnetic clouds at 1 AU. 87, 613. doi $10.1029 /$ JA087iA02p00613

Krall, J.: 2007, Astrophys. J. 657, 559. doi 10.1086/510191.

Krall, J., St. Cyr, O.C.: 2006, Astrophys. J. 652, 1740. doi $10.1086 / 508337$

Kuperus, M., Raadu, M.A.: 1974, Astron. Astrophys. 31, 189.

Lemen, J.R., Title, A.M., Akin, D.J., Boerner, P.F., Chou, C., Drake, J.F., Duncan, D.W., Edwards, C.G., Friedlaender, F.M., Heyman, G.F., Hurlburt, N.E., Katz, N.L., Kushner, G.D., Levay, M., Lindgren, R.W., Mathur, D.P., McFeaters, E.L., Mitchell, S., Rehse, R.A., Schrijver, C.J., Springer, L.A., Stern, R.A., Tarbell, T.D., Wuelser, J.-P., Wolfson, C.J., Yanari, C., Bookbinder, J.A., Cheimets, P.N., Caldwell, D., Deluca, E.E., Gates, R., Golub, L., Park, S., Podgorski, W.A., Bush, R.I., Scherrer, P.H., Gummin, M.A., Smith, P., Auker, G., Jerram, P., Pool, P., Soufli, R., Windt, D.L., Beardsley, S., Clapp, M., Lang, J., Waltham, N.: 2012, Solar Phys. 275, 17. doi 10.1007/s11207-011-9776-8

Lepping, R.P., Burlaga, L.F., Jones, J.A.: 1990, J. Geophys. Res. 95, 11957. doi $10.1029 /$ JA095iA08p11957

Low, B.C., Hundhausen, J.R.: 1995, Astrophys. J. 443, 818. doi 10.1086/175572

Lugaz, N., Downs, C., Shibata, K., Roussev, I.I., Asai, A., Gombosi, T.I.: 2011, Astrophys. J. 738, 127. doi 10.1088/0004-637X/738/2/127

Lynch, B.J., Antiochos, S.K., MacNeice, P.J., Zurbuchen, T.H., Fisk, L.A.: 2004, Astrophys. J. 617, 589. doi $10.1086 / 424564$

Lynch, B.J., Antiochos, S.K., DeVore, C.R., Luhmann, J.G., Zurbuchen, T.H.: 2008, Astrophys. J. 683, 1192. doi:10.1086/589738

Lynch, B.J., Antiochos, S.K., Li, Y., Luhmann, J.G., DeVore, C.R.: 2009, Astrophys. J. 697, 1918. doi:10.1088/0004-637X/697/2/1918

Ma, S., Raymond, J.C., Golub, L., Lin, J., Chen, H., Grigis, P., Testa, P., Long, D.: 2011, Astrophys. J. 738, 160. doi 10.1088/0004-637X/738/2/160

Manchester, W.B. IV, Vourlidas, A., Tóth, G., Lugaz, N., Roussev, I.I., Sokolov, I.V., Gombosi, T.I., De Zeeuw, D.L., Opher, M.: 2008, Astrophys. J. 684, 1448. doi $10.1086 / 590231$. 
Munro, R.H., Gosling, J.T., Hildner, E., MacQueen, R.M., Poland, A.I., Ross, C.L.: 1979, Solar Phys. 61, 201. doi 10.1007/BF00155456

Nisticò, G., Bothmer, V., Patsourakos, S., Zimbardo, G.: 2010, Annales Geophysicae 28, 687. doi 10.5194/angeo-28-687-2010

Ontiveros, V., Vourlidas, A.: 2009, Astrophys. J. 693, 267. doi 10.1088/0004-637X/693/1/267

Pariat, E., Antiochos, S.K., DeVore, C.R.: 2009, Astrophys. J. 691, 61. doi $10.1088 / 0004-$ $637 X / 691 / 1 / 61$

Patsourakos, S., Vourlidas, A., Stenborg, G.: 2010, Astrophys. J. Lett. 724, L188. doi $10.1088 / 2041-8205 / 724 / 2 /$ L188

Patsourakos, S., Pariat, E., Vourlidas, A., Antiochos, S.K., Wuelser, J.P.: 2008, Astrophys. J. Lett. 680, L73. doi $10.1086 / 589769$

Plunkett, S.P., Vourlidas, A., Simberová, S., Karlický, M., Kotrč, P., Heinzel, P., Kupryakov, Y.A., Guo, W.P., Wu, S.T.: 2000, Solar Phys. 194, 371.

Régnier, S., Walsh, R.W., Alexander, C.E.: 2011, Astron. Astrophys. 533, L1. doi $10.1051 / 0004-6361 / 201117381$

Robbrecht, E., Patsourakos, S., Vourlidas, A.: 2009, Astrophys. J. 701, 283. doi $10.1088 / 0004-$ $637 \mathrm{X} / 701 / 1 / 283$

Rouillard, A.P.: 2011, Journal of Atmospheric and Solar-Terrestrial Physics 73, 1201. doi $10.1016 /$ j.jastp.2010.08.015

Roussev, I.I., Lugaz, N., Sokolov, I.V.: 2008, In: G. Li, Q. Hu, O. Verkhoglyadova, G. P. Zank, R. P. Lin, \& J. G. Luhmann (ed.) American Institute of Physics Conference Series, American Institute of Physics Conference Series 1039, 286. doi 10.1063/1.2982460

Schwenn, R.: 2006, Living Reviews in Solar Physics 3, 2.

Sheeley, N.R., Hakala, W.N., Wang, Y.-M.: 2000, J. Geophys. Res. 105, 5081. doi $10.1029 / 1999 J A 000338$

Sheeley, N.R. Jr., Wang, Y.-M.: 2007, Astrophys. J. 655, 1142. doi $10.1086 / 510323$

Sime, D.G., Hundhausen, A.J.: 1987, J. Geophys. Res. 92, 1049. doi $10.1029 /$ JA092iA02p01049

Stenborg, G., Vourlidas, A., Howard, R.A.: 2008, Astrophys. J. 674, 1201. doi $10.1086 / 525556$

Thernisien, A., Vourlidas, A., Howard, R.A.: 2009, Forward Modeling of Coronal Mass Ejections Using STEREO/SECCHI Data. 256, 111. doi 10.1007/s11207-009-9346-5

Thernisien, A., Vourlidas, A., Howard, R.A.: 2011, Journal of Atmospheric and SolarTerrestrial Physics 73, 1156. doi 10.1016/j.jastp.2010.10.019

Thernisien, A.F.R., Howard, R.A., Vourlidas, A.: 2006, Modeling of Flux Rope Coronal Mass Ejections. 652, 763. doi $10.1086 / 508254$

Vourlidas, A., Bemporad, A.: 2012, A Decade of Coronagraphic and Spectroscopic Studies of CME-Driven Shocks, American Institute of Physics Conference Series, in print.

Vourlidas, A., Ontiveros, V.: 2009, In: X. Ao \& G. Z. R. Burrows (ed.) American Institute of Physics Conference Series, American Institute of Physics Conference Series 1183, 139. doi $10.1063 / 1.3266770$

Vourlidas, A., Wu, S.T., Wang, A.H., Subramanian, P., Howard, R.A.: 2003, Astrophys. J. 598, 1392. doi $10.1086 / 379098$

Vourlidas, A., Howard, R.A., Esfandiari, E., Patsourakos, S., Yashiro, S., Michalek, G.: 2010, Astrophys. J. 722, 1522. doi $10.1088 / 0004-637 \mathrm{X} / 722 / 2 / 1522$.

Wagner, W.J.: 1984, Ann. Rev. Astron. Astrophys. 22, 267. doi $10.1146 /$ annurev.aa.22.090184.001411.

Wang, Y.-M., Sheeley, N.R. Jr.: 2006, Astrophys. J. 650, 1172. doi 10.1086/506611

Wang, Y.-M., Stenborg, G.: 2010, Astrophys. J. Lett. 719, L181. doi 10.1088/20418205/719/2/L181

Wood, B.E., Karovska, M., Chen, J., Brueckner, G.E., Cook, J.W., Howard, R.A.: 1999, Astrophys. J. 512, 484. doi $10.1086 / 306758$

Wood, B.E., Wu, C.-C., Howard, R.A., Socker, D.G., Rouillard, A.P.: 2011, Astrophys. J. 729, 70. doi $10.1088 / 0004-637 X / 729 / 1 / 70$.

Xie, H., Ofman, L., Lawrence, G.: 2004, Journal of Geophysical Research (Space Physics) 109, 3109. doi $10.1029 / 2003 J A 010226$

Xue, X.H., Wang, C.B., Dou, X.K.: 2005, Journal of Geophysical Research (Space Physics) 110, 8103. doi 10.1029/2004JA010698.

Yan, Y., Pick, M., Wang, M., Krucker, S., Vourlidas, A.: 2006, Solar Phys. 239, 277. doi $10.1007 / \mathrm{s} 11207-006-0202-6$ 
Zhao, X.P., Plunkett, S.P., Liu, W.: 2002, Journal of Geophysical Research (Space Physics) 107, 1223. doi 10.1029/2001JA009143.

Zuccarello, F.P., Bemporad, A., Jacobs, C., Mierla, M., Poedts, S., Zuccarello, F.: 2012, Astrophys. J. 744, 66. doi $10.1088 / 0004-637 \mathrm{X} / 744 / 1 / 66$ 